\title{
Model Predictive Control for Connected Hybrid Electric Vehicles
}

\author{
Kaijiang Yu, ${ }^{1}$ Xiaozhuo Xu, ${ }^{1}$ Qing Liang, ${ }^{2}$ Zhiguo Hu, ${ }^{1}$ Junqi Yang, \\ Yanan Guo, ${ }^{3}$ and Hongwei Zhang ${ }^{1}$ \\ ${ }^{1}$ School of Electrical Engineering and Automation, Henan Polytechnic University, Jiaozuo 454000, China \\ ${ }^{2}$ College of Computer Science and Technology, Henan Polytechnic University, Jiaozuo 454000, China \\ ${ }^{3}$ Graduate School of Integrated Frontier Sciences, Kyushu University, 744 Motooka, Nishi-ku, Fukuoka 8190395, Japan
}

Correspondence should be addressed to Kaijiang Yu; yu.kaijiang@163.com

Received 25 May 2015; Revised 12 July 2015; Accepted 21 July 2015

Academic Editor: Xiaosong Hu

Copyright (C) 2015 Kaijiang Yu et al. This is an open access article distributed under the Creative Commons Attribution License, which permits unrestricted use, distribution, and reproduction in any medium, provided the original work is properly cited.

\begin{abstract}
This paper presents a new model predictive control system for connected hybrid electric vehicles to improve fuel economy. The new features of this study are as follows. First, the battery charge and discharge profile and the driving velocity profile are simultaneously optimized. One is energy management for HEV for $P_{\text {batt }}$; the other is for the energy consumption minimizing problem of acc control of two vehicles. Second, a system for connected hybrid electric vehicles has been developed considering varying drag coefficients and the road gradients. Third, the fuel model of a typical hybrid electric vehicle is developed using the maps of the engine efficiency characteristics. Fourth, simulations and analysis (under different parameters, i.e., road conditions, vehicle state of charge, etc.) are conducted to verify the effectiveness of the method to achieve higher fuel efficiency. The model predictive control problem is solved using numerical computation method: continuation and generalized minimum residual method. Computer simulation results reveal improvements in fuel economy using the proposed control method.
\end{abstract}

\section{Introduction}

In recent years, the energy and environmental problems are emphasized. In particular, energy consumption of vehicles accounts for a substantial amount in the transportation sector. There are various approaches to reduce the fuel consumption of vehicles [1-5]. High efficient vehicles are being developed to increase fuel economy using lightweight automobiles, efficient power train systems, electric vehicles, and hybrid vehicles [1]. On the other hand, the socalled ecodriving can also reduce the fuel consumption [59]. Ecodriving can be characterized as avoiding aggressive acceleration or braking at any road-traffic situations, cruising at steady speed, decelerating smoothly at stops with little or no braking, and maintaining an optimal distance from the preceding vehicle. An ecological control of a single vehicle on a road with up-down shapes [2] and efficient spacing control of multiple vehicles [10] were presented.

A lot of works have been published on the energy management problem of hybrid electric vehicle (HEV) and plugin hybrid electric vehicle (PHEV) systems. These approaches are typical in a family of optimal control techniques. They can be subdivided into four categories: numerical optimization, analytical optimal control theories, instantaneous optimization, and heuristic control techniques [11]. The most representative of numerical optimization is dynamic programming (DP) [11, 12]. However DP is based on fixed speed patterns which are impossible to get in reality. A kind of analytical optimal control techniques is Pontryagin's minimum principle [13]. It gives necessary conditions that the optimal solution must satisfy. It also needs to know the entire driving cycle in advance. The convex optimization method [14] is also a kind of analytical optimal control techniques. The global optimality is guaranteed and the optimal solution can be rapidly and efficiently attained by solvers available. The instantaneous optimization includes the equivalent consumption minimization strategy (ECMS) $[2,15]$. It is based on instantaneous optimization and is easy to implement in real-time. However it cannot guarantee the optimality over the whole driving cycle. Heuristic control techniques like rule-based control strategies [2] are robust, but they are impossible to guarantee the optimality. 
To obtain even more fuel economy improvements, connected hybrid electric vehicles can be considered to reduce the air resistance. The air resistance of a vehicle is proportional to the square of the vehicle speed. When a vehicle runs at $100 \mathrm{~km} / \mathrm{h}$, its aerodynamic drag reaches more than sixty percent of total motion resistance forces [16]. It is obvious that its air resistance causes high fuel consumption. However, the air resistance can be reduced by maintaining a short spacing between two vehicles. Connected vehicles in an automated highway system can lead to increased driver safety, decreased road congestion, and improved fuel economy [17]. Connected vehicles can improve fuel economy through reduced wind resistance [18-20].

A low pressure area distributes in the rear of the lead vehicle. The size of the area can be different by changing the spacing between the vehicles. If the following vehicle runs at the back of the lead vehicle with a short spacing, its air resistance is decreased owing to improved airflow profile between the vehicles. Furthermore, the air resistance of the lead vehicle is also decreased by the smooth airflow [20]. Therefore, fuel consumption of both vehicles can be reduced. However, it is difficult to follow the lead vehicle with a short spacing at high speed by a human driver. Automated cruise control of the vehicle should be introduced to achieve this. Various conventional longitudinal control systems have been proposed such as vehicle following method using information of other vehicles [10] and point following method using a certain decided phase point [21]. A control law for internal combustion engine vehicles is proposed which uses relative speed and spacing information from the preceding and following vehicles in order to choose the proper control action for smooth vehicle following and for maintaining a desired intervehicle spacing specified by the driver [22]. Connected automatic guided electric vehicles to solve problems of traffic saturation, relying on GPS sensors and intervehicle communication, are addressed in [23]. However, these conventional methods consider string stability only. The quantitative effect of road shape and air resistance on fuel consumption for hybrid electric vehicles (HEVs) has not been researched.

For connected hybrid electric vehicles, it is necessary to compute the optimum control inputs of the vehicles by anticipating the future situations including road shape, vehicles' states, and road loads. Therefore, model predictive control (MPC) method can be used.

This paper extends HEV energy management research by adding two novel contributions. First, the battery charge and discharge profile and the driving velocity profile are simultaneously optimized. We make the two connected problems together: one is energy management for HEV for the battery; the other is for the energy consumption minimizing problem of speed control of two vehicles. In reality, the two connected problems are coupled together and affect each other always. The speed of the vehicle affects the charge and discharge profile of the battery. The charge and discharge profile of the battery affects the speed of the vehicle. Second, a new policy between the global optimization method and the instantaneous optimization method is developed. The global optimization method like dynamic programming needs all the information in the future to compute the global optimal control input. The instantaneous optimization method needs no information in the future to compute the control input. The easiest way to deal with the complicated control system is to divide the longitudinal vehicle control system into an upper and lower level controller. The upper level controller determines the desired acceleration of the vehicle on the basis of the position and velocity relative to the other vehicles in the string. The lower level controller determines the input commands to the engine and the braking system, to accomplish the desired acceleration. Also, there is possible to consider road slope, wind, and so forth as a disturbance for the problem. However, in this work we intended to optimize the fuel economy and the speed profile for high fuel efficiency and safety simultaneously. In the HEV operation it is desirable to charge or discharge the battery properly according to the road loads. There is a problem between the fast dynamics components like the engine and the slow dynamics components like the battery. The prediction horizon of the battery state is limited. We developed a new policy to predict the battery state in a longer future for better performance. The desired battery state of charge is designed according to the road slopes for better recuperation of free braking energy. The battery state of charge profile is scheduled systematically to improve fuel economy inside the HEV considering the effect of different parameters, that is, road conditions, battery state of charge, and real-time implementation ability. The quantitative analysis of the vehicle spacing influence and the battery state of charge profile influence for the fuel economy is presented. Performance of the proposed system has been evaluated by computer simulation. The proposed system is found to be more fuel efficient and safer for running over several typical roads with up-down slopes.

The rest of this paper is organized as follows. In Section 2, the nonlinear model of two connected power-split HEVs is derived. Section 3 formulates the nonlinear model predictive control algorithm. Section 4 presents comparative simulation results. Section 5 provides conclusions.

\section{Modeling of Two Connected HEVs}

The configuration of the HEV system is shown in Figure 1. FD represents the final drive. The power-split device (PSD) is the key component of the power-split HEV system and has both functionality of speed coupler and continuously variable transmission (CVT). There are five dynamic components: the engine, the battery, two motor/generators $(M / G)$, and the wheels in this power-split HEV system. The only dynamic state to be considered in the optimal control problem based on known driving cycle is the battery state of charge (SOC) which can simplify the MPC algorithm for implementation. This simplification is possible because this paper introduces four constraints: the road load, the torque and speed relationship of the speed coupler, the power flow relationship among the five components, and the engine optimal operating line (OOL) using CVT. In this work, we assume that the engine works along its OOL using CVT. For simplicity, we assume the two vehicle configurations are the same. It is assumed that the central controller set in the lead vehicle controls the two vehicles. The central controller computes the control inputs 


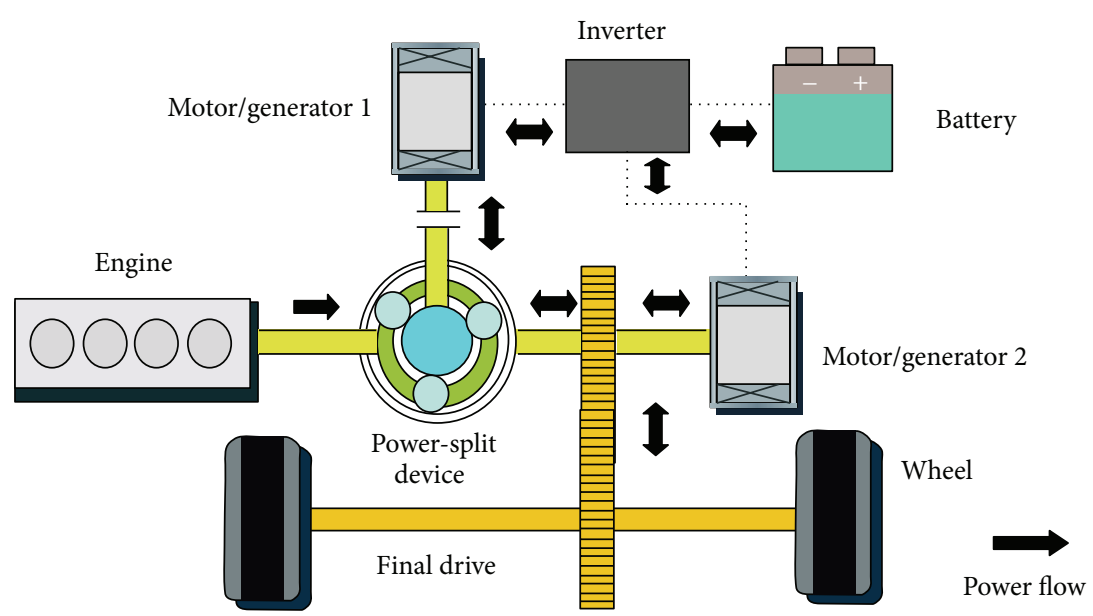

FIGURE 1: Configuration of the power-split HEV system. Diagram adapted from [12].

of the two vehicles. The control inputs of the two vehicles are fed into the two vehicles, respectively. The states of the two vehicles are measured and sent to the central controller. In this way a closed control loop is formed. Here, we call it central control system of connected vehicles. In a distributed control system of connected vehicles [18-20], the individual vehicles are controlled separately by its own controller. It cannot predict other vehicles precisely. In a central control system of connected vehicles, all the information of the vehicles is shared, and the global optimality of all the vehicles can be obtained. The distance between the two vehicles changes (which affect the air drag coefficient) and the slope changes; therefore the stability of this controller is very important. However, the control scheme proposed in this work is brand new; the stability of this controller is completely different from that of the distributed control system of connected vehicles. Hence, we would like to add the stability problem as our future directions because of its complexity. The control signals are transmitted to the vehicles through intervehicle communication. It is assumed that there is no delay of the communication. The proposed methodology will work independently of other kinds of vehicles on the roadway in the network if the vehicle has the functionality of CVT. This paper divided the optimal control problem into two levels. The high-level controller determines the optimal battery power and the low-level controller determines the optimal torque and speed of the engine and the motor/generators. This paper focuses on the high-level controller.

The torque and speed relationship of the speed coupler can be expressed as [24]

$$
\begin{aligned}
& \tau_{\text {eng }}(t)=-\left(1+\frac{R}{S}\right) \tau_{M / G 1}(t), \\
& \tau_{\text {eng }}(t)=-\left(1+\frac{S}{R}\right)\left(\tau_{M / G 2}(t)-\frac{\tau_{\text {req }}(t)}{g_{f}}\right), \\
& S \omega_{M / G 1}(t)+R \omega_{M / G 2}(t)-(S+R) \omega_{\text {eng }}(t)=0,
\end{aligned}
$$

where $S$ and $R$ are the number of sun gear and ring gear teeth, respectively, $\tau_{M / G 1}, \tau_{M / G 2}, \tau_{\text {req }}$, and $\tau_{\text {eng }}$ are the torques of $M / G 1, M / G 2$, the road load, and the engine, respectively, and $\omega_{M / G 1}, \omega_{M / G 2}$, and $\omega_{\text {eng }}$ are the angular speeds of $M / G 1$, $M / G 2$, and the engine, respectively.

The power flow relationships among the five components at the inverter and the power-split device in Figure 1 are given as

$$
\begin{aligned}
& P_{\text {batt }}(t)=P_{M / G 1}(t)+P_{M / G 2}(t), \\
& P_{\text {req }}(t)=P_{M / G 1}(t)+P_{M / G 2}(t)+P_{\text {eng }}(t),
\end{aligned}
$$

where $P_{\text {batt }}, P_{M / G 1}, P_{M / G 2}, P_{\text {eng }}$, and $P_{\text {req }}$ are the power of the battery, $M / G 1, M / G 2$, the engine, and the road load.

This paper assumes that the engine always works along its OOL using CVT which can also be considered as a constraint. When the engine power is known, by looking up the table of OOL, the engine speed and torque can be obtained.

This paper evaluates the fuel consumption using Willans line method to reduce the complexity of the engine fuel consumption model. It was found that good approximations are obtained using the Willans line method [25]. The fuel consumption can be expressed as

$$
\begin{aligned}
\dot{m}_{f}(t) & =\dot{m}_{f}\left(P_{\text {req }}(t)-P_{\text {batt }}(t)\right) \\
& \approx c_{f}\left(P_{\text {req }}(t)-P_{\text {batt }}(t)\right),
\end{aligned}
$$

where $c_{f}$ is a constant. The detailed explanation of this fuel consumption model is included in Appendix A.

The road loads which are the vehicle speed and the required power at the wheels are known when the driving cycle is known. From the configuration of the power-split HEV system, $M / G 2$ speed is also known as

$$
\omega_{M / G 2}(t)=\frac{g_{f}}{r_{w}} v_{\text {req }}(t),
$$

where $\omega_{M / G 2}$ is the speed of $M / G 2, g_{f}$ is the final drive gear ratio, $r_{w}$ is the wheel radius, and $v_{\text {req }}$ is the required vehicle speed by the driving cycle.

For simplicity, it is assumed that there are two cars in this central control system. When the driving cycle is unknown, 
the system dynamics includes the battery and the vehicle dynamics. Both the fuel economy and the driving profile are optimized. The system model is then represented by

$\dot{x}$

$$
\begin{aligned}
& {\left[\begin{array}{c}
z_{p}-\frac{(1 / 2) \rho C_{D p} A_{p} v_{p}^{2}}{m_{p}}-g \mu-g \sin \left(\theta_{p}\left(p_{p}\right)\right) \\
k_{p p}\left(u_{p}-z_{p}\right) \\
-\frac{V_{\text {OC } p}-\sqrt{V_{\text {OC } p}^{2}-4 P_{\text {batt } p} R_{\text {batt } p}}}{2 R_{\text {batt } p} Q_{\text {batt } p}} \\
\left.z_{h}-\frac{(1 / 2) \rho C_{D h} A_{h} v_{h}^{2}-g \mu-g \sin \left(\theta_{h}\right.}{m_{h}}\left(p_{h}\right)\right) \\
k_{p h}\left(u_{h}-z_{h}\right) \\
-\frac{V_{\text {OCh }}-\sqrt{V_{\text {OCh }}^{2}-4 P_{\text {batt } h} R_{\text {batth }}}}{2 R_{\text {batt } h} Q_{\text {batth } h}}
\end{array}\right]} \\
& x=\left[\begin{array}{llllllll}
p_{p} & v_{p} & z_{p} & x_{\mathrm{SOC} p} & z_{h} & v_{h} & w_{h} & x_{\mathrm{SOCh}}
\end{array}\right]^{T} \text {, } \\
& u=\left[\begin{array}{llll}
u_{p} & P_{\text {batt } p} & u_{h} & P_{\text {batth }}
\end{array}\right]^{T} \text {, }
\end{aligned}
$$

where $p, v$, and $z$ are the vehicle position, speed, and acceleration or deceleration converted from the traction force or brake force. The parameters $\rho, C_{D}, A, m, g, \mu$, and $\theta(p)$ are the air density, the air drag coefficient, the frontal area of the vehicle, the vehicle mass, the gravity acceleration, the rolling resistance coefficient, and the road grade. $u_{p}, u_{h}$, and $k_{p}$ are the vehicle acceleration or deceleration control inputs and the delay constant. $V_{\mathrm{OC}}, R_{\text {batt }}$, and $Q_{\text {batt }}$ are the open-circuit voltage, the internal resistance, and the capacity of the battery. The suffixes $p$ and $h$ denote the parameters of the preceding vehicle and the host vehicle.

The slope information from GPS or the digital map is approximated by the sigmoid functions. This modeling method of road slope is firstly proposed in this work. A description of the modeling method is provided in Appendix B.

\section{Model Predictive Control}

The driving control inputs are derived using model predictive control algorithm. The optimal control problem is defined as

$$
\text { Min. } J=\int_{t}^{t+T} L(x(\tau \mid t), u(\tau \mid t)) d \tau
$$

subject to $\mathrm{SOC}_{p \min } \leq x_{\mathrm{SOC} p}(\tau \mid t) \leq \mathrm{SOC}_{p \max }$

$$
\begin{aligned}
& P_{\text {batt } p \min } \leq P_{\text {batt } p}(\tau \mid t) \leq P_{\text {batt } p \max } \\
& u_{p \min } \leq u_{p}(\tau \mid t) \leq u_{\text {max }} \\
& \mathrm{SOC}_{h \min } \leq x_{\text {SOCh }}(\tau \mid t) \leq \mathrm{SOC}_{h \max } \\
& P_{\text {batthmin }} \leq P_{\text {batth }}(\tau \mid t) \leq P_{\text {batt } h \text { max }} \\
& u_{h \min } \leq u_{h}(\tau \mid t) \leq u_{h \max },
\end{aligned}
$$

where $T$ is the prediction horizon and ${ }_{\text {min }}$ and ${ }_{\max }$ denote the minimum and maximum bounds of the parameters.

The following objectives are considered in this optimal control problem.

The term $L_{x}$ : acceleration or deceleration of vehicles is moderated.

The term $L_{y}$ : the vehicle speed is kept near to its desired value.

The term $L_{z}$ : the fuel consumption is minimized.

The term $L_{d}$ : the battery SOC is kept near to its desired value. This is one of the cores of the proposed approach. This paper adapts the battery energy to the vehicle future energy requirements by setting the desired battery SOC as a function of road slopes which represent the main part of the future road load.

The term $L_{e}$ : the battery energy is made best use of. This is one of the cores of the proposed approach. The battery energy is firstly used to satisfy the required road load. If it is not enough, the engine energy should be used, and the engine can work along its OOL.

The term $L_{f}$ : the battery SOC constraint is kept satisfied.

The term $L_{g}$ : the desired vehicle spacing is kept. This is one of the cores of the proposed approach. The following distance constraint is kept in a predictive controller structure. The following distance is varied above the minimum following distance, which improves the freedom of ecodriving car following control to optimize the driving profile for better fuel economy.

The cost function $L$ is defined as follows:

$$
\begin{aligned}
& L=w_{x} L_{x}+w_{y} L_{y}+w_{z} L_{z}+w_{d} L_{d}+w_{e} L_{e}+w_{f} L_{f} \\
& +w_{g} L_{g} \\
& L_{x}=\left(z_{p}-\frac{(1 / 2) \rho C_{D p} A_{p} v_{p}^{2}}{m_{p}}-g \mu\right)^{2} \\
& +\left(z_{h}-\frac{(1 / 2) \rho C_{D h} A_{h} v_{h}^{2}}{m_{h}}-g \mu\right)^{2}, \\
& L_{y}=\left(v_{p}-v_{d}\right)^{2}+\left(v_{h}-v_{d}\right)^{2}, \\
& L_{z}=\frac{c_{f p}\left(m_{p} z_{p} v_{p}-P_{\text {batt } p}\right)}{\left(1+e^{\left(-\beta\left(m_{p} z_{p} v_{p}-P_{\text {batt }}\right)\right)}\right)} \\
& +\frac{c_{f h}\left(m_{h} z_{h} v_{h}-P_{\text {batth }}\right)}{\left(1+e^{\left(-\beta\left(m_{h} z_{h} v_{h}-P_{\text {batth }}\right)\right)}\right)} \\
& L_{d}=\left(x_{\mathrm{SOC} p}-\mathrm{SOC}_{d}\left(p_{p}\right)\right)^{2} \\
& +\left(x_{\mathrm{SOCh}}-\mathrm{SOC}_{d}\left(p_{h}\right)\right)^{2}, \\
& L_{e}=\left(m_{p} w_{p} v_{p}-P_{\text {batt } p}\right)^{2}+\left(m_{h} w_{h} v_{h}-P_{\text {batt } h}\right)^{2},
\end{aligned}
$$




$$
\begin{aligned}
L_{f}= & -\ln \left(x_{\mathrm{SOC} p}-\mathrm{SOC}_{p \min }\right) \\
& -\ln \left(\mathrm{SOC}_{p \max }-x_{\mathrm{SOC} p}\right) \\
& -\ln \left(x_{\mathrm{SOCh}}-\mathrm{SOC}_{h \min }\right) \\
& -\ln \left(\mathrm{SOC}_{h \max }-x_{\mathrm{SOCh}}\right), \\
L_{g}= & \frac{1}{2}\left(d-d_{d}\right)^{2}, \\
d= & p_{p}-p_{h}-l_{p},
\end{aligned}
$$

where $w_{x}, w_{y}, w_{z}, w_{d}, w_{e}, w_{f}$, and $w_{g}$ are the weights and $v_{d}$ is the desired vehicle speed. The parameters $d, d_{d}$, and $l_{p}$ are the vehicle spacing, the desired vehicle spacing, and the length of the preceding vehicle. The parameter $\mathrm{SOC}_{d}$ is the desired SOC value. The sigmoid function is chosen to evaluate the vehicle brake fuel consumption. The log barrier function is used as a penalizing term for violations of state constraints.

The structure of the nonlinear model predictive control system is shown in Figure 2. The system inputs contain the control inputs. The system outputs consist of the vehicle states. The predictive controller uses terrain information from a digital map to calculate $\mathrm{SOC}_{d}(p)$ and $\theta(p)$.

At each time $t$, the optimal control input is computed by solving the above optimal control problems during the prediction horizon $T$. Only the first element of the optimal control sequence is applied. At the next time step, the prediction horizon moves forward, and the process is repeated.

\section{Computer Simulations}

4.1. Comparison Controllers. There are two simulations in this work. They are the MPC approaches with fixed desired battery SOC and variable desired battery SOC. The aim is to demonstrate how the desired battery SOC affects the fuel economy, the power-split profile, and the drag coefficients.

The desired battery SOC value is set according to the road elevation. The authors think it is reasonable to utilize the road elevation information since this future road load information is known already. The desired battery SOC is assumed to use the function as

$$
\begin{aligned}
& \operatorname{SOC}_{d}(p) \\
& =k_{\mathrm{SOC}}\left(\frac{s_{1}}{1+e^{\left(s_{3}\left(p-s_{2}\right)\right)}}+\frac{s_{4}}{1+e^{\left(s_{6}\left(p-s_{5}\right)\right)}}+\cdots\right) \\
& \quad+\operatorname{SOC}_{k},
\end{aligned}
$$

where $k_{\mathrm{SOC}}$ and $\mathrm{SOC}_{k}$ are constant parameters set as $k_{\mathrm{SOC}}=$ -2 and $\mathrm{SOC}_{k}=0.7$, respectively.

4.2. Simulation Conditions. In these simulations, the parameters of both HEVs are used from ADVISOR 2002 Toyota Prius data (see Table 1). Seven tuning weights are used in this cost function; this makes the performance very subjective to choice of these weights. The goal is to minimize total fuel used, so the real cost function should be integral of

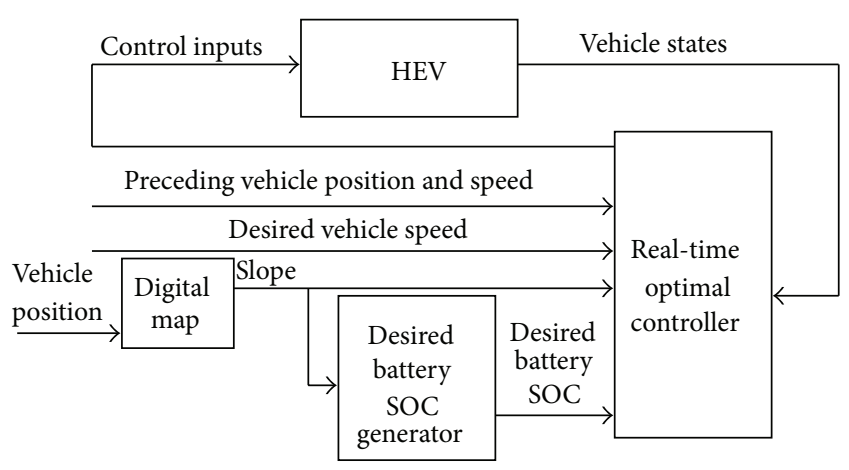

FIGURE 2: Structure of the model predictive control system.

TABLE 1: Simulation parameters.

\begin{tabular}{lc}
\hline Parameters & Values \\
\hline$m$ & 1504 \\
$c_{f}$ & 0.0874 \\
$g$ & $9.8\left[\mathrm{~m} / \mathrm{s}^{2}\right]$ \\
$V_{\mathrm{OC}}$ & $307.9[\mathrm{~V}]$ \\
$Q_{\text {batt }}$ & $6[\mathrm{Ah}]$ \\
$g_{f}$ & 3.93 \\
$l_{p}$ & $4.31[\mathrm{~m}]$ \\
$h_{t}$ & $0.1[\mathrm{~s}]$ \\
SOC $_{\min }$ & 0.6 \\
$w_{x}$ & 100000 \\
$w_{z}$ & 20 \\
$w_{e}$ & 100 \\
$w_{g}$ & 3000 \\
$\rho$ & $1.23\left[\mathrm{~kg} / \mathrm{m}^{3}\right]$ \\
$A$ & $1.746\left[\mathrm{~m}^{2}\right]$ \\
$\mu$ & 0.015 \\
$R_{\text {batt }}$ & $1.0[\Omega]$ \\
$r_{w}$ & $0.287[\mathrm{~m}]$ \\
$k_{p}$ & 10 \\
$d_{d}$ & $1[\mathrm{~m}]$ \\
$\mathrm{SOC}_{d}$ & 0.7 \\
$\mathrm{SOC}_{\max }$ & 0.8 \\
$w_{y}$ & 2000 \\
$w_{d}$ & 67000000 \\
$w_{f}$ & 200000 \\
\hline
\end{tabular}

fuel rate plus an equivalent fuel cost at the end of the MPC horizon. Any other choice makes the cost function very subjective and the optimal controller will not minimize fuel use. Tuning of the weight parameters is an important issue for attaining fuel efficient and safe behavior in the complex system. Weight parameters are tuned manually by observing the fuel economy and driving performance. $w_{x}$ is tuned for minimal fuel consumption by dynamic acceleration of the vehicle. $w_{z}$ is tuned for minimal fuel consumption by static maps of the engine which cannot evaluate the dynamic fuel consumption of the engine. $w_{y}$ and $w_{g}$ are tuned for safety of driving to avoid real-ends collision. $w_{d}$ and $w_{e}$ are tuned 


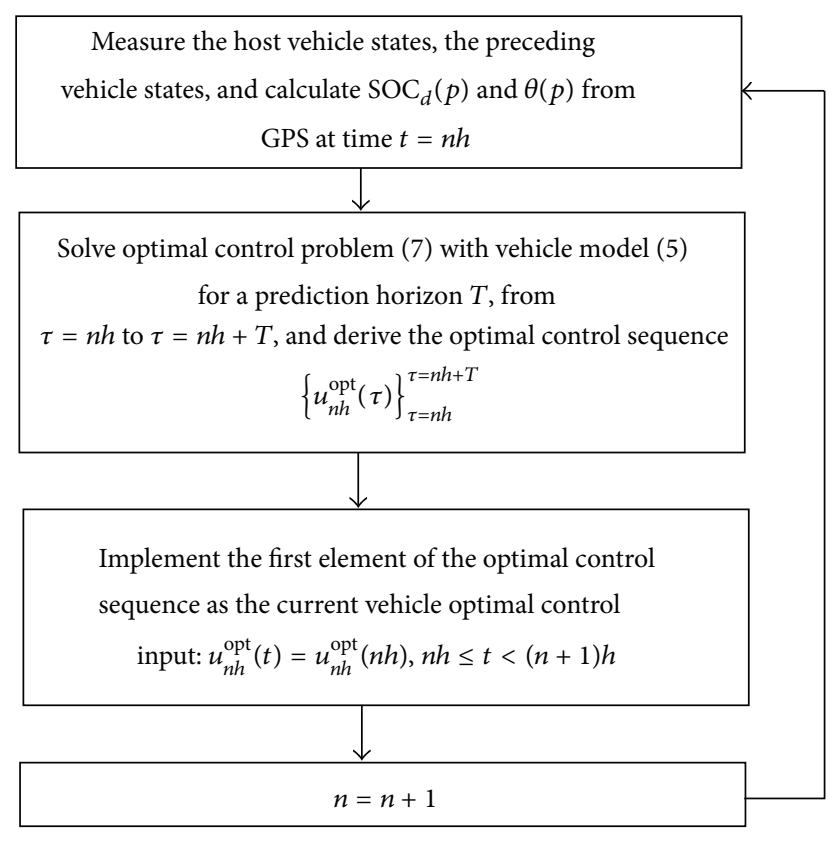

FIGURE 3: Flowchart of the nonlinear real-time optimal control algorithm.

for minimizing the equivalent fuel cost. $w_{f}$ does not need to be tuned ordinarily. The reason is that it is for the state constraint. In reality, it is a physical constraint which cannot be violated.

The model predictive control problem is solved using the numerical computation method: the continuation and generalized minimum residual (C/GMRES) method [26]. The C/GMRES method uses forward difference approach and discretizes the HEV plant with a sampling interval $h_{t}$ to implement the nonlinear real-time optimal control algorithm. A brief description of the solution of the model predictive control problem using the C/GMRES method is included in Appendix C. The flowchart of the nonlinear model predictive control algorithm implementation is shown in Figure 3. Since the optimization problem is nonlinear and nonconvex, its solution can be local optimal; it is assumed that it can only be solved numerically. A detailed mathematical analysis of robust stability and performance for the proposed method can be found in [26]. The fuel economy is calculated using the engine fuel consumption map which is obtained from ADVISOR 2002.

The MPC algorithm is realized by utilizing the C MEX S-function builder in MATLAB/Simulink. First, the optimal battery power is calculated by the high-level controller. Next, this optimal value is fed into the low-level controller where the optimal torque and speed of the engine and $M / G$ s are determined. Finally, these actual control input signals are applied to the vehicle. The fuel economy is calculated using the quasi-static map of ADVISOR. The backward simulation approach has been employed in this work, as ADVISOR software based on quasi-static maps of power train elements is utilized. However, (3) presents a different formula for fuel consumption estimation which is not consistent with the way
TABLE 2: Comparison of the energy used by motion resistance forces.

\begin{tabular}{lc}
\hline$c_{11}$ & $4.498 \times 10^{-8}$ \\
$c_{12}$ & $-1.475 \times 10^{-6}$ \\
$c_{13}$ & $1.139 \times 10^{-5}$ \\
$c_{14}$ & $9.373 \times 10^{-5}$ \\
$c_{15}$ & -0.002 \\
$c_{16}$ & 0.003 \\
$c_{17}$ & 0.035 \\
$c_{18}$ & 0.205 \\
$c_{21}$ & $1.326 \times 10^{-7}$ \\
$c_{22}$ & $-6.593 \times 10^{-6}$ \\
$c_{23}$ & 0.0001 \\
$c_{24}$ & -0.0014 \\
$c_{25}$ & 0.0080 \\
$c_{26}$ & -0.026 \\
$c_{27}$ & 0.046 \\
$c_{28}$ & 0.244 \\
\hline
\end{tabular}

ADVISOR calculates the fuel consumption. The reason is that (3) is for the control input calculation of the model predictive control which needs to be continuous and have derivative, and quasi-static maps of power train elements are used for the output evaluation.

The parameters predicted are the road slope based on the GPS data and the traffic conditions. They are not embedded in ADVISOR, as in this software the slope is assumed to be zero. Quasi-static maps of power train elements in ADVISOR are used only for the output evaluation. The traffic conditions and their impact on the fuel consumption are predicted using the connected vehicle model to achieve optimal vehicle spacing for reducing air drag.

A set of data representing the relationship of the aerodynamic drag coefficient and the spacing obtained from a wind tunnel experiment [20] is adopted. By using the seventh-degree polynomial representation, the aerodynamic drag coefficient $C_{i}$ can be represented as

$$
\begin{aligned}
C_{i}(d(t))= & c_{i 1} d^{7}(t)+c_{i 2} d^{6}(t)+c_{i 3} d^{5}(t)+c_{i 4} d^{4}(t) \\
& +c_{i 5} d^{3}(t)+c_{i 6} d^{2}(t)+c_{i 7} d(t)+c_{i 8},
\end{aligned}
$$

where parameters $c_{i 1}-c_{i 8}$ are shown in Table 2 . The approximation results of $C_{i}$ are shown in Figure 4.

4.3. Simulation Results. The driving profile of the HEV using the MPC algorithm with fixed desired battery SOC and unfixed desired battery SOC (see Figures 5 and 6) shows that the MPC algorithm can use the road slope information well to reduce the fuel consumption. The rows of Figures 5 and 6 from the top are the slope of the road, the speed of the preceding vehicle and the host vehicle, the battery SOC of the preceding vehicle and the host vehicle, and the vehicle distance between the preceding vehicle and the host vehicle. The MPC algorithm simultaneously controls both vehicles by predicting their states, and fast convergence of their spacing is achieved. The vehicle accelerates before the up slope to make use of the kinetic energy. The battery 
TABLE 3: Fuel economy comparison results.

\begin{tabular}{lccc}
\hline Method & $\begin{array}{c}\text { Preceding vehicle } \\
\text { mileage }(\mathrm{km} / \mathrm{L})\end{array}$ & $\begin{array}{c}\text { Host vehicle } \\
\text { mileage }(\mathrm{km} / \mathrm{L})\end{array}$ & $\begin{array}{c}\text { Total } \\
\text { mileage }(\mathrm{km} / \mathrm{L})\end{array}$ \\
\hline MPC with unfixed SOC & $31.9(+2.9 \%)$ & $20.4(+3.0 \%)$ & $12.4(+2.5 \%)$ \\
MPC with fixed SOC & 31.0 & 19.8 & 12.1 \\
\hline
\end{tabular}

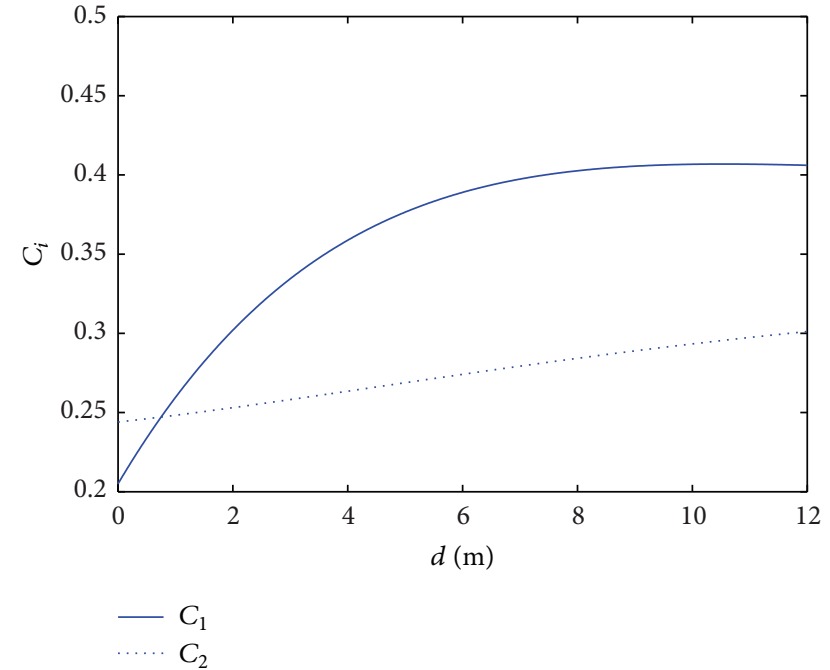

Figure 4: Approximation of $C_{1}$ and $C_{2}$ with respect to the spacing d.

recuperates vehicle braking power during the vehicle down slope driving. The preceding vehicle SOC variation range is smaller than that of the host vehicle. The host vehicle SOC variation range with unfixed desired battery SOC is smaller than that with fixed desired battery SOC. Since the engine needs to charge the battery too often, the overuse of the battery leads to worse fuel economy.

The energy profile of the HEV using the MPC algorithm with fixed desired battery SOC and unfixed desired battery SOC (see Figures 7 and 8) shows that the MPC algorithm can make the vehicle drag coefficients converge to minimum. The rows of Figures 7 and 8 from the top are the air drag power of the preceding vehicle and the host vehicle, the drag coefficients of the preceding vehicle and the host vehicle, the fuel consumption rate of the preceding vehicle and the host vehicle, and the total cumulative fuel consumption. The drag coefficients of both vehicles are reduced significantly as a result of the fast convergence of the vehicle distance. Therefore, the drag force is reduced, and fuel savings are achieved. The air drag power of the host vehicle is smaller than that of the preceding vehicle because of the vehicle platooning. The fuel consumption of the HEV with unfixed desired battery SOC is smaller than that with fixed desired battery SOC. The reason is that, during the down slope driving, the MPC algorithm with unfixed desired battery SOC makes better use of the motor regenerative braking than that with fixed desired battery SOC.

All the constraints are satisfied in the simulation. The overall fuel economy results are presented in Table 3 . It is
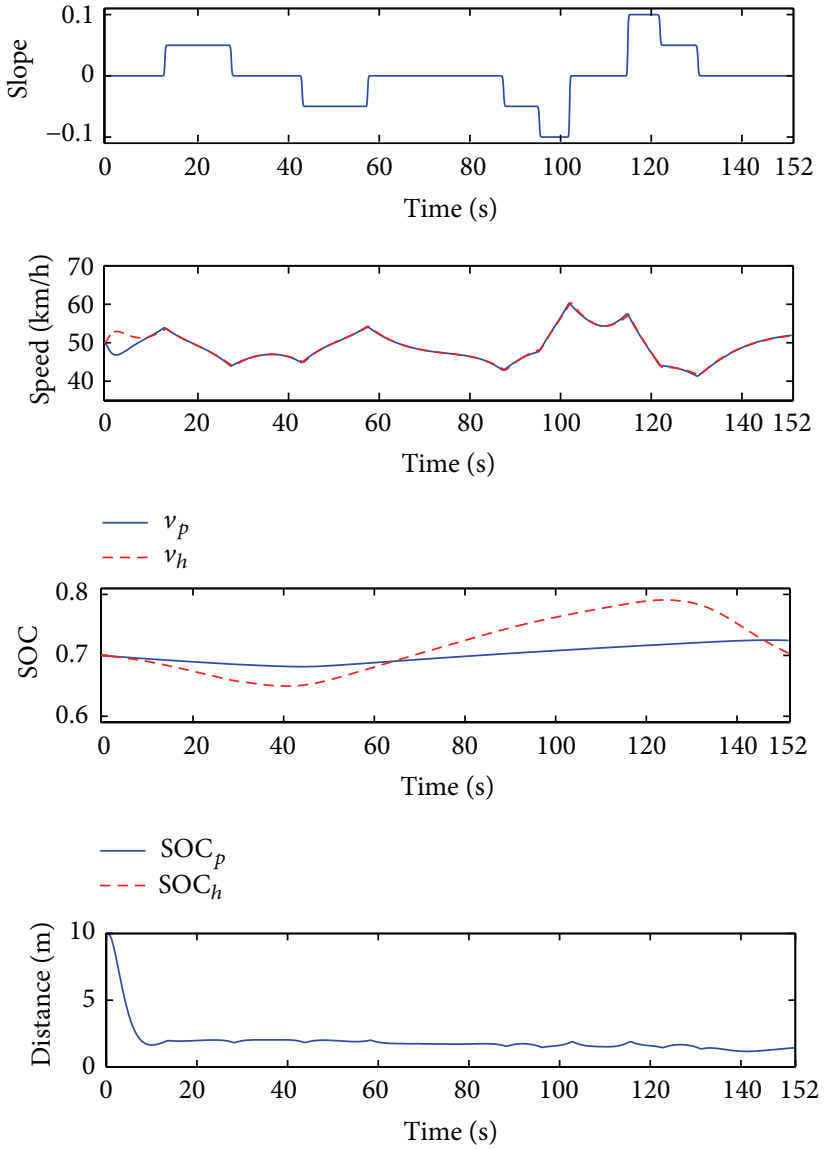

FIGURE 5: Driving profile of the HEV using the MPC algorithm with fixed desired battery SOC.

shown that the MPC approach with unfixed desired battery SOC can improve fuel economy and keep the final SOC near the initial SOC compared to that with fixed desired battery SOC. There are two reasons. The first reason is that the MPC approach with unfixed desired battery SOC makes the battery use less often than that with fixed desired battery SOC. The second reason is that the MPC approach with unfixed desired battery SOC reduces the duration and magnitude of the braking during the down slope driving period compared with that with fixed desired battery SOC. To sum up, the reason why MPC approach improves the fuel economy while keeping the SOC near initial status is the variation of the battery SOC. This variation can facilitate charging when the vehicles decelerate. The proposed method can improve the fuel economy of both the preceding vehicle and the host vehicle. 

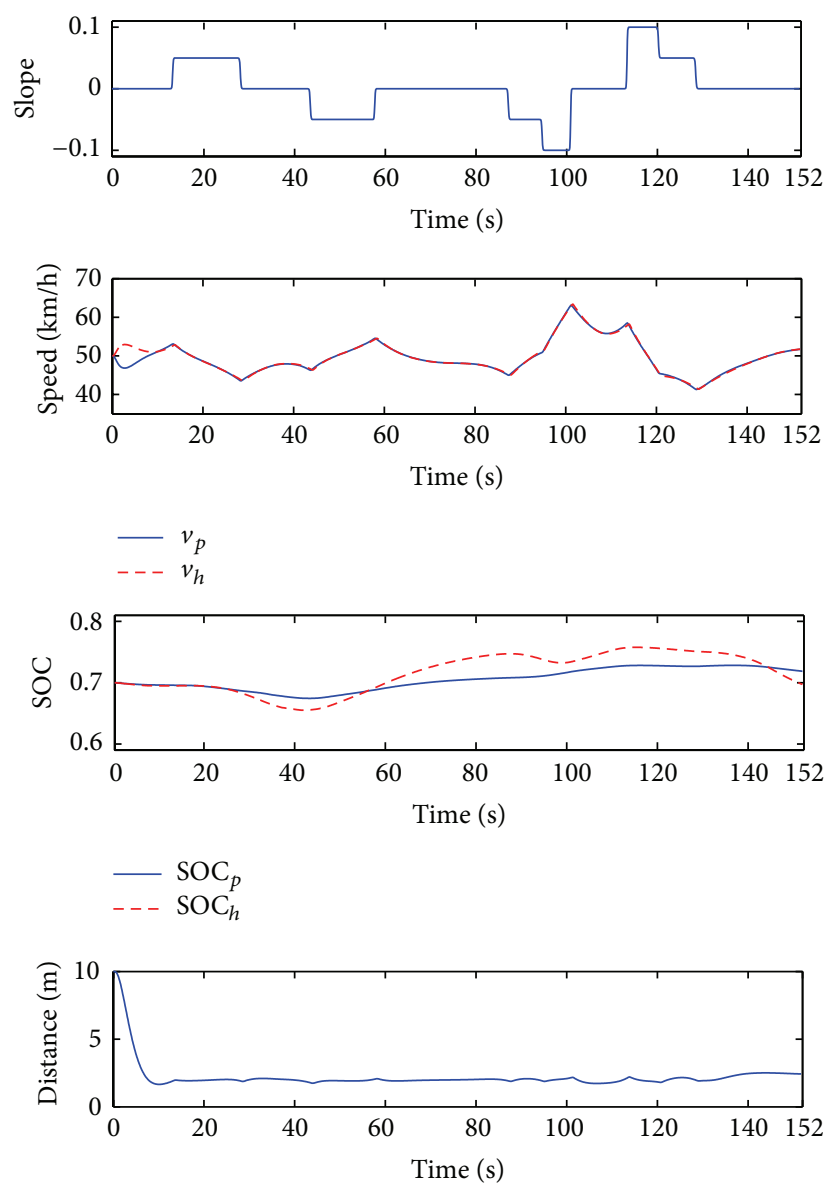

FIGURE 6: Driving profile of the HEV using the MPC algorithm with unfixed desired battery SOC.

The fuel economies with different desired vehicle speed, prediction horizon, and control horizon using the proposed method were shown in Figures 10, 11, and 12. It is shown that the best fuel economy occurs when the desired vehicle speed is equal to $50 \mathrm{~km} / \mathrm{h}$, the prediction horizon is equal to $2 \mathrm{~s}$, and the control horizon is equal to $0.2 \mathrm{~s}$.

MPC is sensitive to process-model mismatch. Processmodel mismatch is always present: wheels, weather and road conditions, sensor accuracy, and so on. Therefore performance and stability under nominal conditions do not guarantee the robust performance and stability of the real car. In order to analyze the robust stability and performance of the MPC method, errors in slope sensor are assumed to exist while traveling. The proposed MPC method is found to be very robust against slope-sensing error. Figure 9 shows the deviation of the driving and power-split profiles due to errors in slope sensor for the lead vehicle. An error of $20 \%$ means that the sensor provides 1.2 times the actual value, whereas $-20 \%$ error means that the sensor provides 0.8 times the actual value of the slope. Due to a sensing error of $20 \%$, the vehicle speed is a bit lower; the battery SOC is a bit higher; and compared with the vehicle with no slope-sensing error, fuel savings dropped 3\%. Similarly, due to a sensing error of $-20 \%$, the vehicle speed is a bit higher; the battery SOC is a bit lower; and compared with the vehicle with no slope-sensing error, fuel savings increased $0.8 \%$. Therefore, it can be concluded that, within a reasonable sensing error, the system is robust to maintain its ecological performance without significant deviation. Since the optimization problem is nonlinear and nonconvex, its solution can be local optimal; it is assumed that it can only be solved numerically. A detailed mathematical analysis of robust stability and performance for the proposed method can be found in [26].

The process-model mismatch can also give rise to important unfeasibility issues when computing the MPC control law. A policy to recover from unfeasibility issues is needed to be developed. The numerical computation method cannot manage unfeasibility by itself. Since we are dealing here with vehicles (i.e., people), a policy to recover from unfeasibility issues cannot be developed by reformulating the MPC problem with soft constraints. A switch policy is proposed to cope with the unfeasibility issues. It is summarized as follows.

Step 1. Switch the MPC algorithm to the rule-based algorithm [1] when unfeasibility issues occur.

Step 2. Use the rule-based algorithm for $5 \mathrm{~s}$, and switch back to the MPC algorithm.

Step 3. Go back to Step 1 when unfeasibility issues occur. 

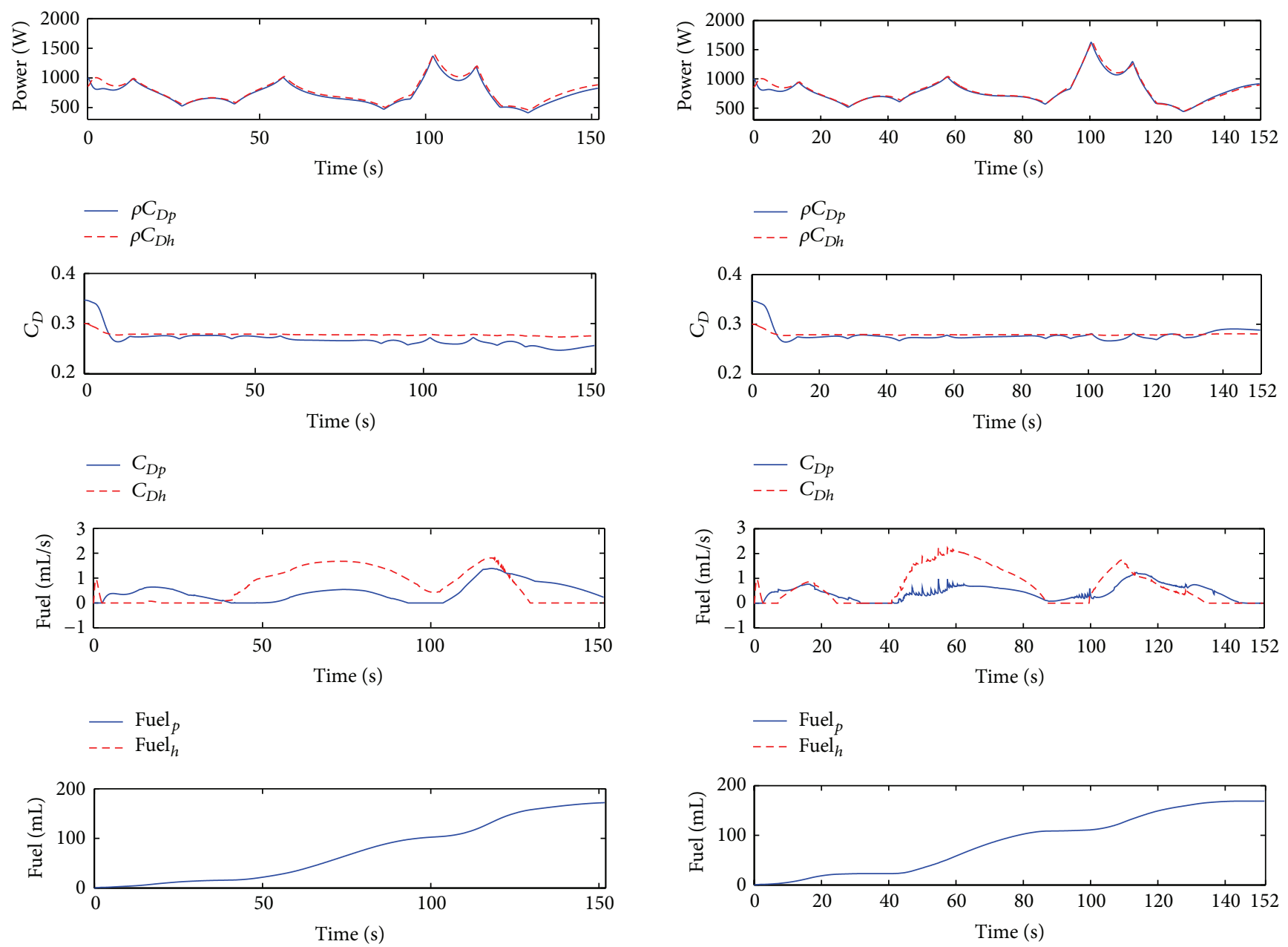

FIGURE 7: Energy profile of the HEV using the MPC algorithm with fixed desired battery SOC.

The proposed MPC algorithm is fast for computation. The computer simulation time is 153 [s]. The computation time of the proposed MPC algorithm is 14 [s]. The simulation is run in a MATLAB/Simulink environment using a laptop with an Intel processor at $2.27[\mathrm{GHz}]$ processing speed and 2 [GB] of RAM. The sampling interval is 100 [ms]. The computation time per sampling interval of the proposed MPC algorithm is 9 [ms]. So it is concluded that the MPC algorithm has the potential for real-time vehicle control.

\section{Conclusions}

A model predictive control system for two connected powersplit HEVs considering the fuel economy, the aerodynamic drag varied by vehicle spacing, and the road shape information has been presented. The performance of the proposed control system was confirmed by the computer simulations. The proposed control method has produced the fast convergence of the vehicle spacing. The excessive acceleration and deceleration have been avoided by predicting the road shapes. The results revealed improvements of the fuel economy considering the effect of different parameters, that is, road conditions, battery state of charge, and real-time implementation

FIGURE 8: Energy profile of the HEV using the MPC algorithm with unfixed desired battery SOC.

ability. Since experiments of vehicles are expensive, we would like to conduct experiments in the future. For the sake of simplicity, we have considered only two cars. In the future, we will add more vehicles to make the model more realistic.

\section{Appendices}

\section{A. Engine Fuel Consumption Model}

The proposed engine fuel consumption modeling method is a special method using both Willans line method and the assumption of operating the engine along the engine optimal operating line and is introduced as follows. The HEV parameters are used from the ADVISOR 2002 Toyota Prius HEV data [27].

The Willans line model consists of an affine representation relating the available energy, that is, the energy that is theoretically available for conversion, to the useful energy that is actually present at the output of the energy converter [25]. Formally

$$
W_{\text {out }}=e W_{\text {in }}-W_{\text {loss }},
$$




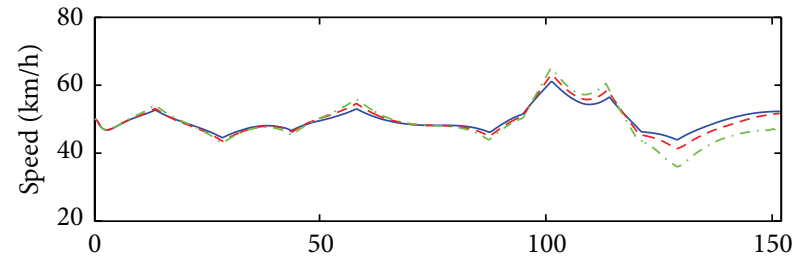

$-20 \%$ slope error

- - $0 \%$ slope error

-..- $20 \%$ slope error

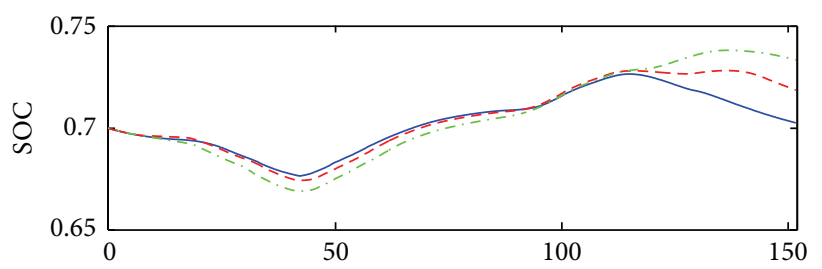

$-20 \%$ slope error

- $-0 \%$ slope error

-. - $20 \%$ slope error

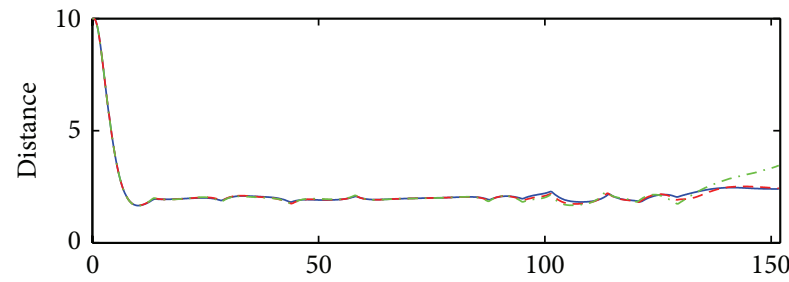

$-20 \%$ slope error
$---0 \%$ slope error
.$--20 \%$ slope error

FIGURE 9: Driving and power-split profile deviation due to the slope-sensing error.

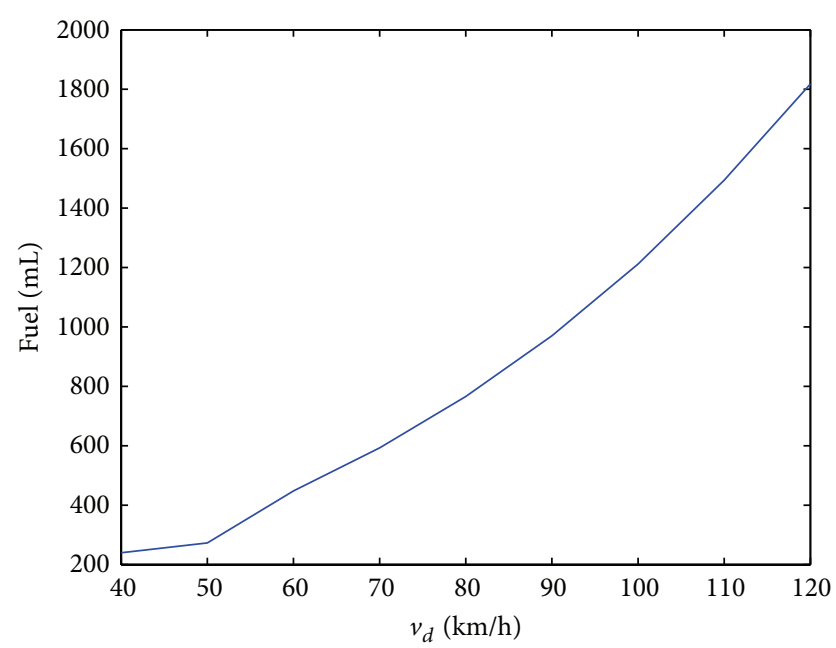

FIGURE 10: Effects of the desired vehicle speed on fuel economy.

where the parameter $e$ represents the peak intrinsic energy conversion efficiency of the converter and $W_{\text {loss }}$ represents external (parasitic) losses. In fact, this model of energy

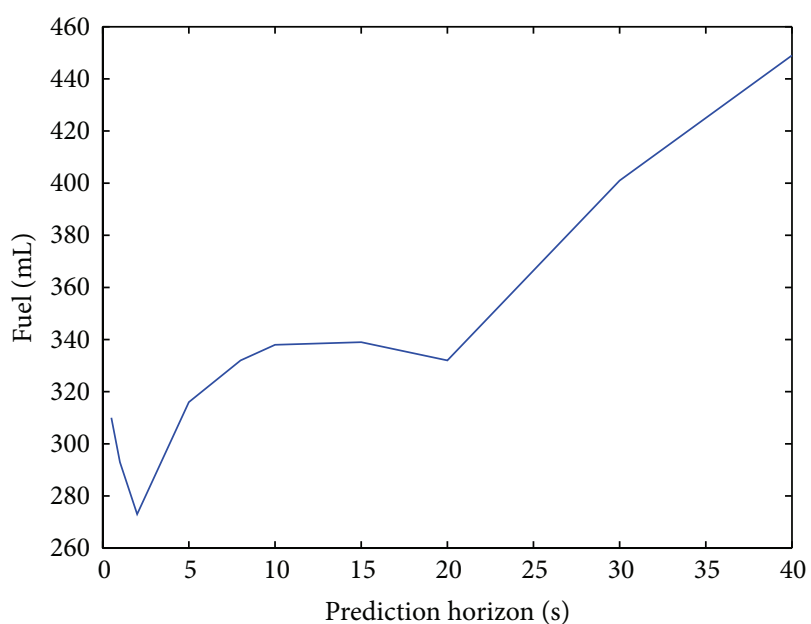

FIGURE 11: Effects of the prediction horizon on fuel economy.

conversion efficiency is nonlinear, in that the parameters $e$ and $W_{\text {loss }}$ are represented as explicit functions of the output flow variable (e.g., engine speed) and are also implicit functions of the effort variable. 


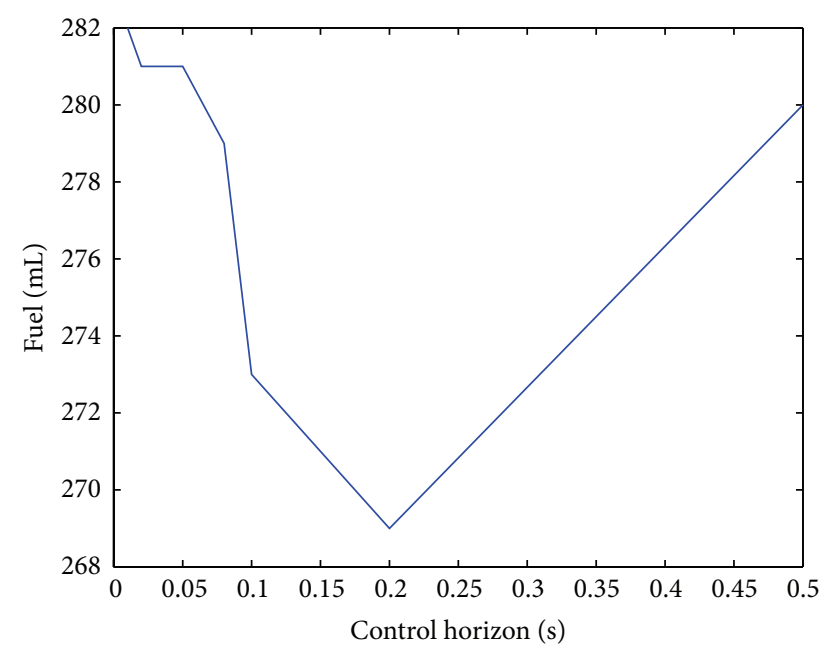

FiguRE 12: Effects of the control horizon on fuel economy.

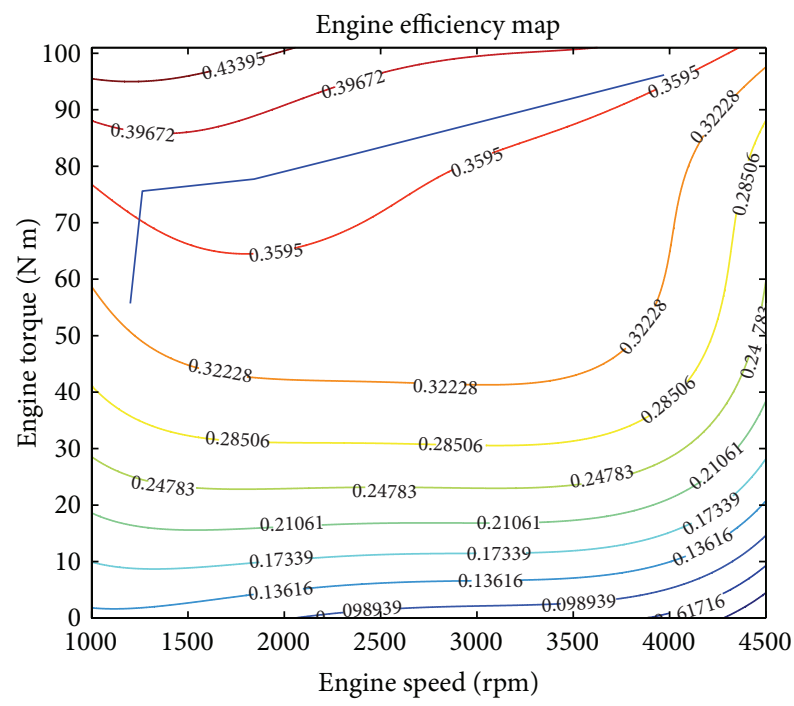

FIGURE 13: The engine efficiency map to the best engine operating points.

The modeling method given above is for general engines. However, in this work, the electric CVT can realize idle stop, so $W_{\text {loss }}$ becomes zero. When it is assumed that the engine operating points are maintained at the best efficiency, the parameters $e$ can be approximated as a constant. In this case, the fuel consumption rate corresponding to the optimal operating line can be fitted using a linear function.

The engine optimal operating line can be plotted on the engine map as shown in Figure 13. The engine optimal operating points provide the highest efficiency for a given power level. The engine best efficiency related to the engine power according to the engine characteristics is shown in Figure 14.

The fuel consumption rate is estimated as (see Figure 15)

$$
\dot{m}_{f}=\frac{P_{\mathrm{eng}}}{C \eta} \approx c_{f} P_{\mathrm{eng}},
$$

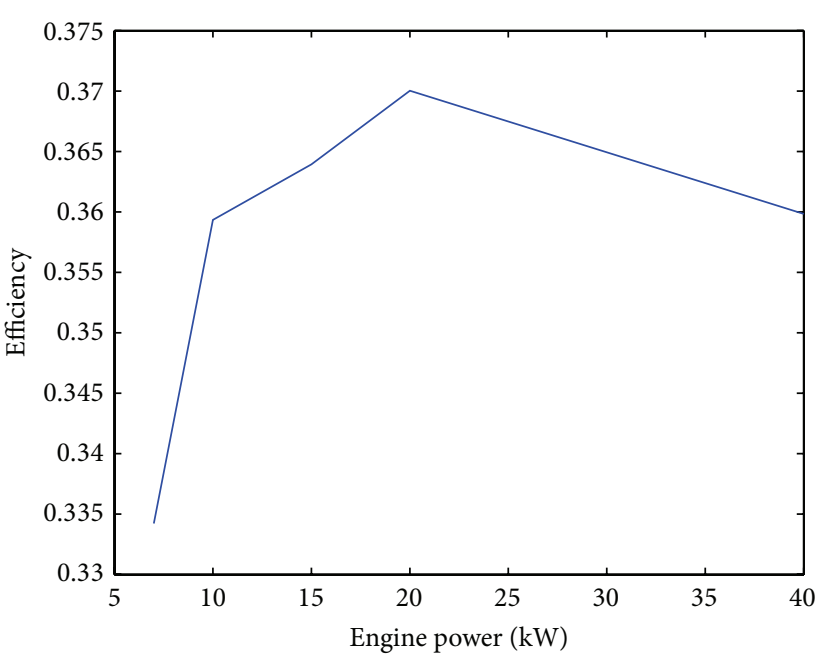

FIGURE 14: The engine efficiency curve to the best engine operating points.

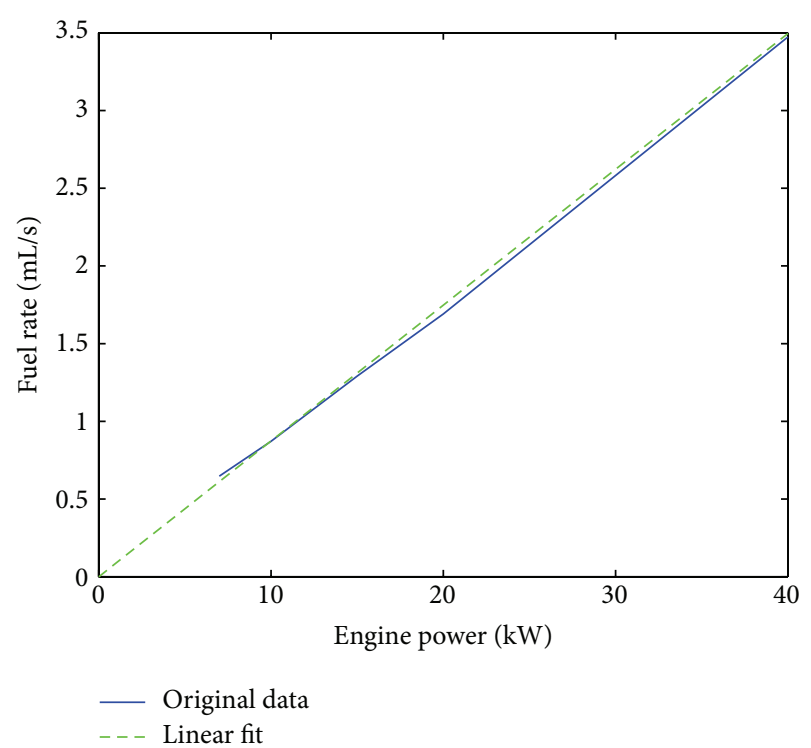

FIGURE 15: The engine fuel consumption rate to the best engine operating points.

where $C$ is the calorific value of the gasoline, which is equal to $34.5 \times 10^{6}[\mathrm{~J} / \mathrm{l}]$, and $\eta$ is the engine efficiency.

\section{B. Road Slope Modeling Method}

A brief description of the road slope modeling method is provided as follows. In this research, the sigmoid function is used to model the road slope. The general sigmoid function to model the road slope is expressed as follows:

$$
\theta(p)=\frac{s_{1}}{1+e^{\left(s_{3}\left(p-s_{2}\right)\right)}}+\frac{s_{4}}{1+e^{\left(s_{6}\left(p-s_{5}\right)\right)}}+\cdots,
$$

where $s_{1}, s_{2}, s_{3}, s_{4}, s_{5}$, and $s_{6}$ are slope shape parameters. The parameters $s_{2}, s_{5}, \ldots$ are position parameters where the 

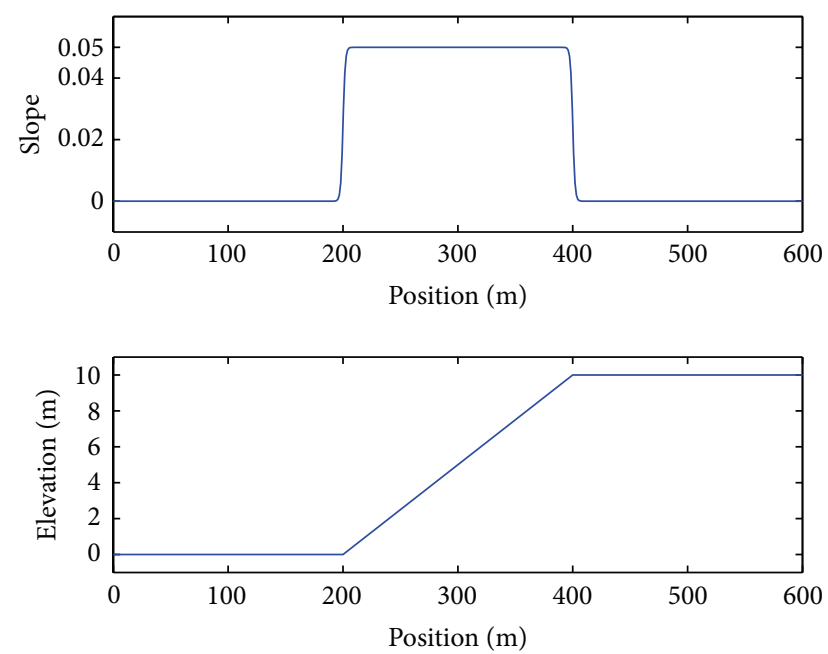

FIGURE 16: The sigmoid function of the up slope.
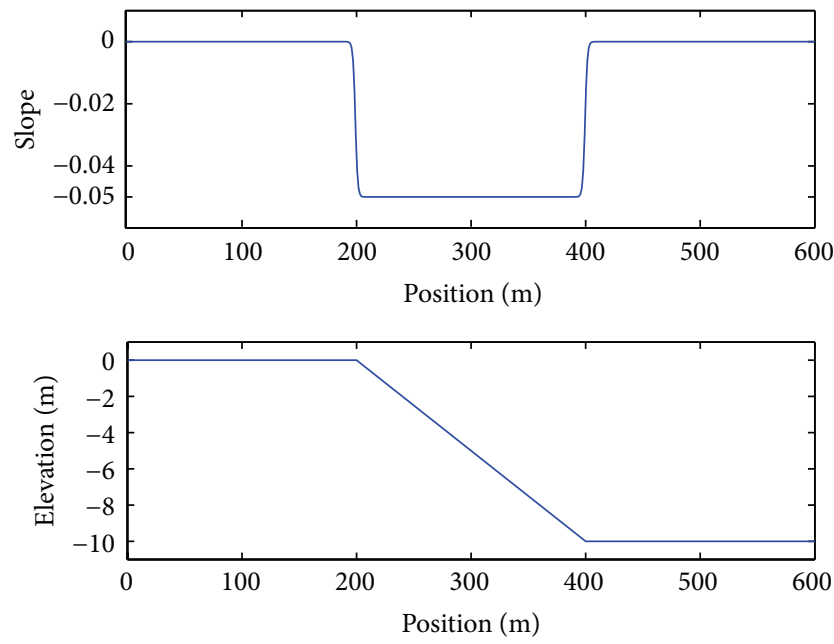

FIgURE 17: The sigmoid function of the down slope.

road slopes change. The parameters $s_{3}, s_{6}, \ldots$ are parameters to decide the abruptness of the road slope.

When it is the up slope case, the sigmoid function is expressed as follows:

$$
\theta(p)=\frac{0.05}{1+e^{(-(p-200))}}+\frac{-0.05}{1+e^{(-(p-400))}}
$$

The figure of the sigmoid function is showed in Figure 16.

When it is the down slope case, the sigmoid function is expressed as follows:

$$
\theta(p)=\frac{-0.05}{1+e^{(-(p-200))}}+\frac{0.05}{1+e^{(-(p-400))}}
$$

The figure of the sigmoid function is showed in Figure 17.
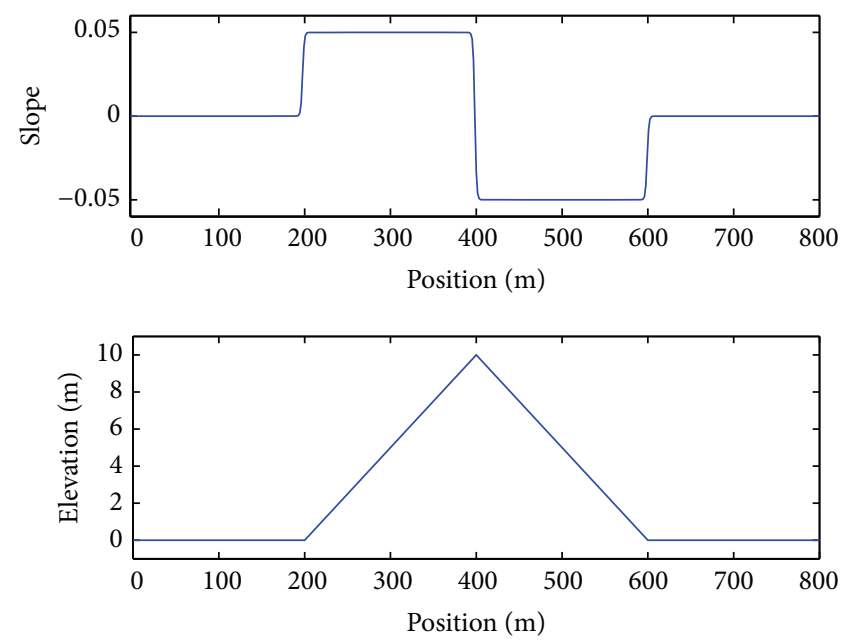

FIGURE 18: The sigmoid function of the up-down slope.

When it is the up-down slope case, the sigmoid function is expressed as follows:

$$
\begin{aligned}
\theta(p)= & \frac{0.05}{1+e^{(-(p-200))}}+\frac{-0.1}{1+e^{(-(p-400))}} \\
& +\frac{0.05}{1+e^{(-(p-600))}}
\end{aligned}
$$

The figure of the sigmoid function is showed in Figure 18.

\section{Solution of the Model Predictive Control Problem}

A brief description of the solution of the model predictive control problem is provided as follows.

To implement the model predictive control algorithm, the horizon $T$ is divided into $N$ steps, and the optimal control problem is discretized. The general discretized optimal control problem is formulated as

$$
\min _{u} J=\sum_{i=0}^{N-1} L\left(x_{i}(\tau \mid t), u_{i}(\tau \mid t)\right) \Delta \tau(t)
$$

$$
\begin{array}{ll}
\text { subject to } \quad & x_{i+1}(\tau \mid t) \\
& =x_{i}(\tau \mid t) \\
& +f\left(x_{i}(\tau \mid t), u_{i}(\tau \mid t)\right) \Delta \tau(t) \\
& G\left(x_{i}(\tau \mid t), u_{i}(\tau \mid t)\right) \leq 0,
\end{array}
$$

where $u$ is the control input, $x$ is the state, and $L$ is the cost function. $f(x, u)$ is the state equation. $G(x, u)$ is the inequality constraint.

The inequality constraint in the optimal control problem is converted to an equality constraint by introducing a dummy input $u_{d}$ for computation simplicity as follows:

$$
C(x(t), u(t))=u^{2}(t)+u_{d}^{2}(t)-u_{\max }^{2}=0,
$$

where $u_{\max }$ denotes the upper bound of the control input. 
To solve this optimal control problem with the calculus of variation method, the Hamiltonian function is defined by

$$
H(x, u, \lambda, \psi)=L(x, u)+\lambda^{T} f(x, u)+\psi^{T} C(x, u),
$$

where $\lambda$ denotes the costate and $\psi$ denotes the Lagrange multiplier associated with the equality constraint.

The first-order necessary conditions for the optimal control input $u$, the multiplier $\psi$, and the costate $\lambda$ are obtained using the calculus of variation as

$$
\begin{aligned}
& x_{i+1}(t)=x_{i}(t)+f\left(x_{i}(t), u_{i}(t)\right) \Delta \tau(t) \\
& x_{0}(t)=x(t), \\
& \lambda_{i}(t) \\
& =\lambda_{i+1}(t) \\
& +H_{x}\left(x_{i}(t), u_{i}(t), \lambda_{i+1}(t), \psi_{i}(t)\right) \Delta \tau(t) \\
& \lambda_{N}(t)=0, \\
& H_{u}\left(x_{i}(t), u_{i}(t), \lambda_{i+1}(t), \psi_{i}(t)\right)=0 \\
& C(x(t), u(t))=0,
\end{aligned}
$$

where $x_{0}$ is the initial state.

To solve this optimal control problem, the continuation and GMRES (C/GMRES) method is employed for computation cost reduction. The necessary conditions of optimality for the constrained control input can be expressed as the following equation:

$$
\begin{aligned}
& F(U(\tau \mid t), x(\tau \mid t), t) \\
& :=\left[\begin{array}{c}
H_{u}\left(u_{0}(\tau \mid t), x_{0}(\tau \mid t), \lambda_{1}(\tau \mid t), \psi_{0}(\tau \mid t)\right) \\
C\left(u_{0}(\tau \mid t), x_{0}(\tau \mid t)\right) \\
\vdots \\
H_{u}\left(u_{N-1}(\tau \mid t), x_{N-1}(\tau \mid t), \lambda_{N}(\tau \mid t), \psi_{N-1}(\tau \mid t)\right) \\
C\left(u_{N-1}(\tau \mid t), x_{N-1}(\tau \mid t)\right)
\end{array}\right] \\
& =0 \text {, } \\
& U(t) \\
& :=\left[u_{0}^{T}(\tau \mid t), \psi_{0}^{T}(\tau \mid t), \ldots, u_{N-1}^{T}(\tau \mid t), \psi_{N-1}^{T}(\tau \mid t)\right]^{T} . \\
& F(U(t), x(t), t)=0 \text { is identical to } \\
& F(U(0), x(0), 0):=0, \\
& \dot{F}(U, x, t)=-A_{s} F(U(t), x(t), t),
\end{aligned}
$$

where $A_{s}$ is a stable matrix introduced to stabilize $F=0$. If $F_{U}$ is nonsingular, a differential equation for $U(t)$ can be obtained as

$$
\dot{U}=-F_{U}^{-1}\left(A_{s} F-F_{x} \dot{x}-F_{t}\right) .
$$

The above differential equation can be solved by the GMRES method. The presented approach is also a kind of continuation method. The solution curve $U(t)$ is traced by integrating

the above differential equation. Because there is no need to calculate the Jacobians and the linear equation iteratively, C/GMRES method assures the real-time optimal control ability because of small computational cost. The detailed description of the solution for the model predictive control algorithm can be found [26].

\section{Notations and Abbreviations}

HEVs:

MPC:

CVT:

OOL:

SOC:

PSD:

$M / G$ :

FD:

A:

$C_{D}$ :

$g:$

$g_{f}:$

$m:$

$P_{\text {batt: }}$ :

$P_{\text {eng }}$ :

$Q_{\text {batt: }}$ :

$r_{w}$ :

$R_{\text {batt: }}$ :

$V_{\text {OC }}$ :

$\theta$ :

$\mu:$

$\rho:$

S:

$R:$

$\tau_{M / G 1}$ :

$\tau_{M / G 2}$ :

$\tau_{\text {req }}$ :

$\tau_{\text {eng }}:$

$\omega_{M / G 1}$ :

$\omega_{M / G 2}$ :

$\omega_{\text {eng }}$ :

$P_{\text {req: }}$ :

$c_{f}$ :

$p$ :

$v:$

$w:$

$u_{p}:$

$u_{h}$ :

$k_{p}$ :

$s_{1}, s_{2}, s_{3}, s_{4}, s_{5}$, and $s_{6}$ :

$T$ :

$\min$ and ${ }_{\text {max }}$ :

$w_{x}, w_{y}, w_{z}, w_{d}$,

$w_{e}, w_{f}$, and $w_{g}$ : The weights

Final drive

Frontal area brake force
Hybrid electric vehicles

Model predictive control

Continuously variable transmission

Optimal operating line

State of charge

Power-split device

Motor/generator

Aerodynamic drag coefficient

Gravitational acceleration

Final drive gear ratio

Vehicle mass

Battery power

Engine power

Battery nominal capacity

Wheel radius

Battery internal resistance

Battery open-circuit voltage

Road inclination

Rolling friction coefficient

Density of air

The number of sun gear teeth

The number of ring gear teeth

The torque of motor/generator 1

The torque of motor/generator 2

The torque of the road load

The torque of the engine

The angular speed of motor/generator 1

The angular speed of motor/generator 2

The angular speed of the engine

The power of the road load

A constant for the fuel consumption

The vehicle position

The vehicle speed

The acceleration or deceleration

converted from the traction force or

The acceleration or deceleration control input of the preceding vehicle

The acceleration or deceleration control input of the host vehicle

The delay constant

Slope shape parameters

The prediction horizon

The minimum and maximum bounds of the parameters 


$\begin{array}{ll}v_{d}: & \text { The desired vehicle speed } \\ d: & \text { The vehicle spacing } \\ d_{d}: & \text { The desired vehicle spacing } \\ l_{p}: & \text { The length of the preceding vehicle } \\ \text { SOC }_{d}: & \text { The desired SOC value } \\ k_{\mathrm{SOC}} \text { and } \mathrm{SOC}_{k}: & \text { Constant parameters } \\ c_{i 1}-c_{i 8}: & \text { Constant parameters for the } \\ C: & \text { aerodynamic drag coefficients } \\ u: & \text { The calorific value of the gasoline } \\ x: & \text { The control input } \\ L: & \text { The state } \\ f(x, u): & \text { The state equation } \\ G(x, u): & \text { The inequality constraint } \\ u_{d}: & \text { The dummy input } \\ u_{\mathrm{max}}: & \text { The upper bound of the control input } \\ \lambda: & \text { The costate } \\ \psi: & \text { The Lagrange multiplier associated with } \\ & \text { the equality constraint } \\ x_{0}: & \text { The initial state } \\ A_{s}: & \text { A stable matrix. }\end{array}$

\section{Acknowledgments}

This project is supported by the National Natural Science Foundation of China (Grant nos. 51405137, 61403129), the Key Scientific Research Program of the Higher Education Institutions of Henan Province (Grant no. 15A470014), the Science and Technology Planning Project of Jiaozuo City in 2014 (Grant no. 2014110013), and the Doctoral Program Foundation of Henan Polytechnic University (Grant no. 60807/010).

\section{Conflict of Interests}

The authors declare that there is no conflict of interests regarding the publication of this paper.

\section{References}

[1] A. Sciarretta and L. Guzzella, "Control of hybrid electric vehicles," IEEE Control Systems Magazine, vol. 27, no. 2, pp. 60-70, 2007.

[2] C. Zhang, A. Vahidi, P. Pisu, X. Li, and K. Tennant, "Role of terrain preview in energy management of hybrid electric vehicles," IEEE Transactions on Vehicular Technology, vol. 59, no. 3, pp. 1139-1147, 2010.

[3] K. Yu and J. Yang, "Performance of a nonlinear real-time optimal control system for HEV s/PHEV during car following," Journal of Applied Mathematics, vol. 2014, Article ID 879232, 14 pages, 2014.

[4] K. Yu, H. Yang, Q. Liang, J. Yang, and Y. Guo, "Model predictive control for hybrid electric vehicle platooning using route information," Proceedings of the Institution of Mechanical Engineers Part D: Journal of Automobile Engineering. In press.

[5] K. Yu, M. Mukai, and T. Kawabe, "Model predictive control of a power-split hybrid electric vehicle system," Artificial Life and Robotics, vol. 17, no. 2, pp. 221-226, 2012.
[6] K. Yu, M. Mukai, and T. Kawabe, "A battery management system using nonlinear model predictive control for a hybrid electric vehicle," in Proceedings of the 7th IFAC Symposium on Advances in Automotive Control, pp. 301-306, Tokyo, Japan, September 2013.

[7] K. Yu, J. Yang, and D. Yamaguchi, "Model predictive control for hybrid vehicle ecological driving using traffic signal and road slope information," Control Theory and Technology, vol. 13, no. 1, pp. 333-344, 2015.

[8] K. Yu, Q. Liang, Z. Hu, J. Yang, and H. Zhang, "Performance of an eco-driving model predictive control system for HEVs during car following," Asian Journal of Control, 2015.

[9] K. Yu, Q. Liang, J. Yang, and Y. Guo, "Model predictive control for hybrid electric vehicle platooning using slope information," IEEE Transactions on Intelligent Transportation Systems. In press.

[10] D. Swaroop, J. K. Hedrick, C. C. Chien, and P. Ioannou, "A comparision of spacing and headway control laws for automatically controlled vehicles," Vehicle System Dynamics, vol. 23, no. 8, pp. 597-625, 1994.

[11] L. Serrao, A comparative analysis of energy management strategies for hybrid electric vehicles [Ph.D. thesis], The Ohio State University, Columbus, Ohio, USA, 2009.

[12] J. Liu and H. Peng, "Modeling and control of a power-split hybrid vehicle," IEEE Transactions on Control Systems Technology, vol. 16, no. 6, pp. 1242-1251, 2008.

[13] N. Kim, S. Cha, and H. Peng, "Optimal control of hybrid electric vehicles based on Pontryagin's minimum principle," IEEE Transactions on Control Systems Technology, vol. 19, no. 5, pp. 1279-1287, 2011.

[14] X. Hu, N. Murgovski, L. M. Johannesson, and B. Egardt, "Comparison of three electrochemical energy buffers applied to a hybrid bus powertrain with simultaneous optimal sizing and energy management," IEEE Transactions on Intelligent Transportation Systems, vol. 15, no. 3, pp. 1193-1205, 2014.

[15] C. Musardo, G. Rizzoni, Y. Guezennec, and B. Staccia, "AECMS: an adaptive algorithm for hybrid electric vehicle energy management," European Journal of Control, vol. 11, no. 4-5, pp. 509-524, 2005.

[16] H. Yamazaki and K. Okamoto, "Fuel saving by trucks in singlefile platooning on the expressway," JARI Research Journal, vol. 32, no. 3, pp. 139-143, 2010.

[17] R. Rajamani, H.-S. Tan, B. K. Law, and W.-B. Zhang, "Demonstration of integrated longitudinal and lateral control for the operation of automated vehicles in platoons," IEEE Transactions on Control Systems Technology, vol. 8, no. 4, pp. 695-708, 2000.

[18] M. Barth, "An emissions and energy comparison between a simulated automated highway system and current traffic conditions," in Proceedings of the IEEE Intelligent Transportation Systems Proceedings, Dearborn, Mich, USA, October 2000.

[19] Y. Yamamura, Y. Seto, and M. Nagai, "Study on a string-stable ACC using vehicle-to-vehicle communication (2nd report, ACC design method using model predictive control)," Transactions of the Japan Society of Mechanical Engineers Series $C$, vol. 73, no. 7, pp. 1917-1922, 2007.

[20] M. Zabat, N. Stabile, S. Frascaroli, and F. Browand, The Aerodynamic Performance of Platoons: A Final Report, California Partners for Advanced Transit and Highways, 1995.

[21] R. E. Fenton and R. J. Mayhan, "Automated highway studies at the Ohio State University-an overview," IEEE Transactions on Vehicular Technology, vol. 40, no. 1, pp. 100-113, 1991. 
[22] Y. Zhang, E. B. Kosmatopoulos, P. A. Ioannou, and C. C. Chien, "Autonomous intelligent cruise control using front and back information for tight vehicle following maneuvers," IEEE Transactions on Vehicular Technology, vol. 48, no. 1, pp. 319-328, 1999.

[23] J. Bom, B. Thuilot, F. Marmoiton, and P. Martinet, "Nonlinear control for urban vehicles platooning, relying upon a unique kinematic GPS," in Proceedings of the IEEE International Conference on Robotics and Automation (ICRA '05), pp. 4138-4143, IEEE, April 2005.

[24] M. Ehsani, Y. Gao, and A. Emadi, Modern Electric, Hybrid Electric, and Fuel Cell Vehicles: Fundamentals, Theory, and Design, CRC, Boca Raton, Fla, USA, 2nd edition, 2009.

[25] G. Rizzoni, L. Guzzella, and B. M. Baumann, "Unified modeling of hybrid electric vehicle drivetrains," IEEE/ASME Transactions on Mechatronics, vol. 4, no. 3, pp. 246-257, 1999.

[26] T. Ohtsuka, "A continuation/GMRES method for fast computation of nonlinear receding horizon control," Automatica, vol. 40, no. 4, pp. 563-574, 2004.

[27] K. B. Wipke, M. R. Cuddy, and S. D. Burch, "ADVISOR 2.1: a user-friendly advanced powertrain simulation using a combined backward/forward approach," IEEE Transactions on Vehicular Technology, vol. 48, no. 6, pp. 1751-1761, 1999. 


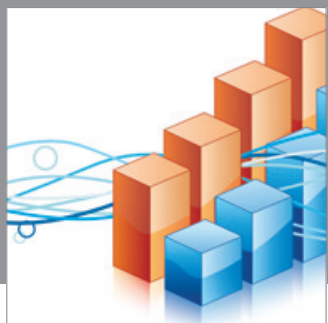

Advances in

Operations Research

mansans

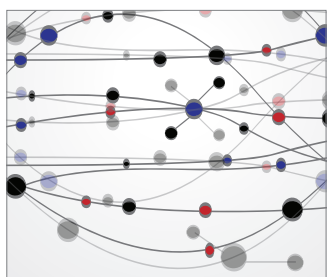

The Scientific World Journal
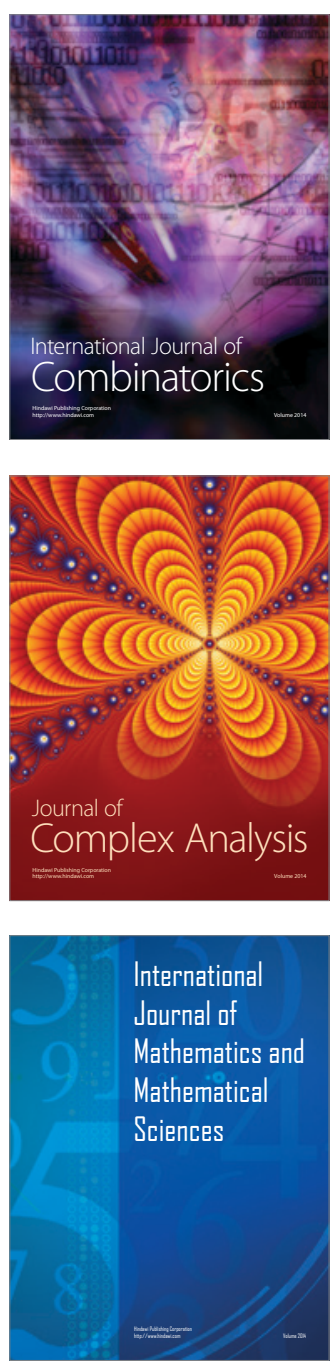
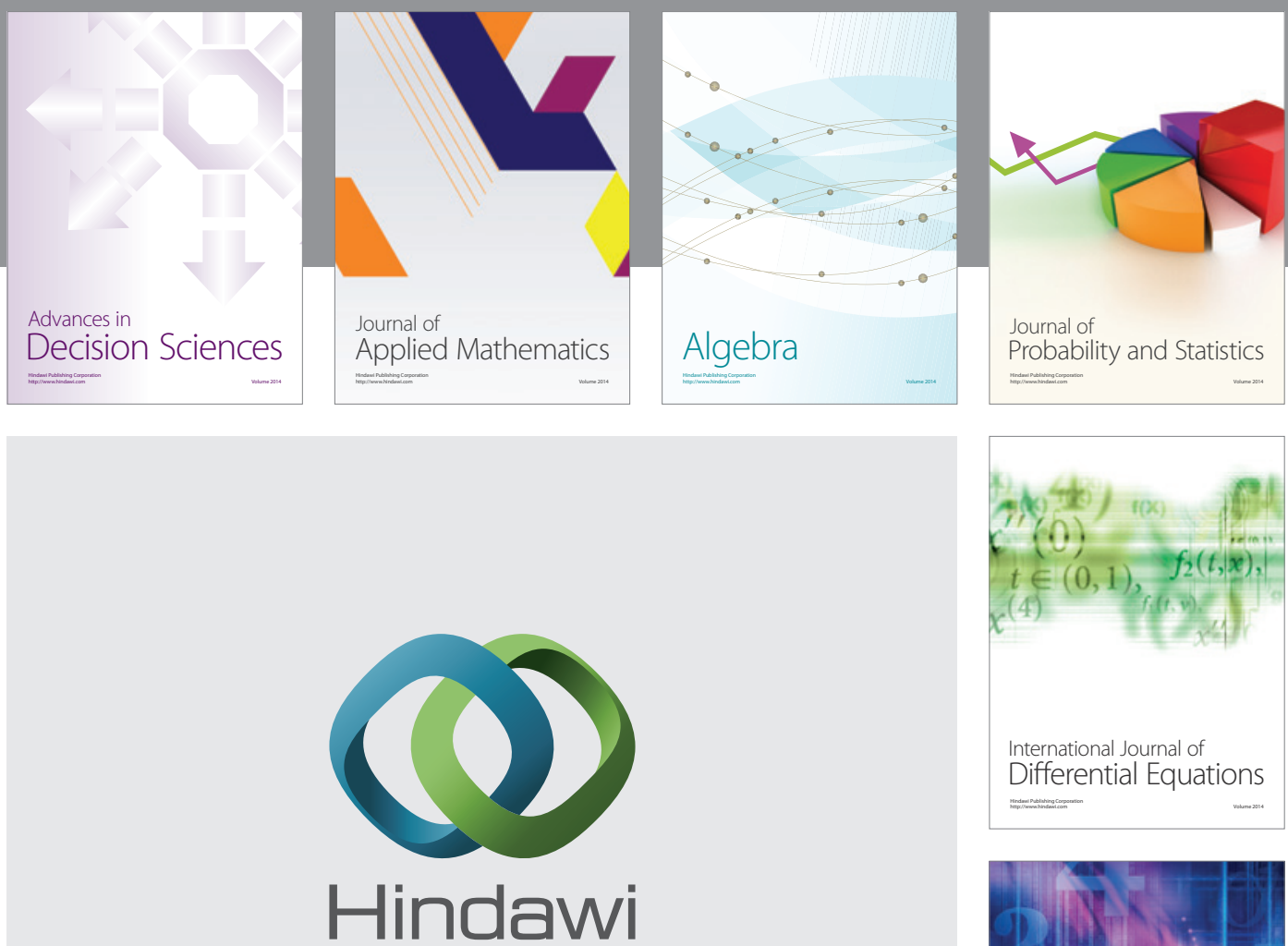

Submit your manuscripts at http://www.hindawi.com
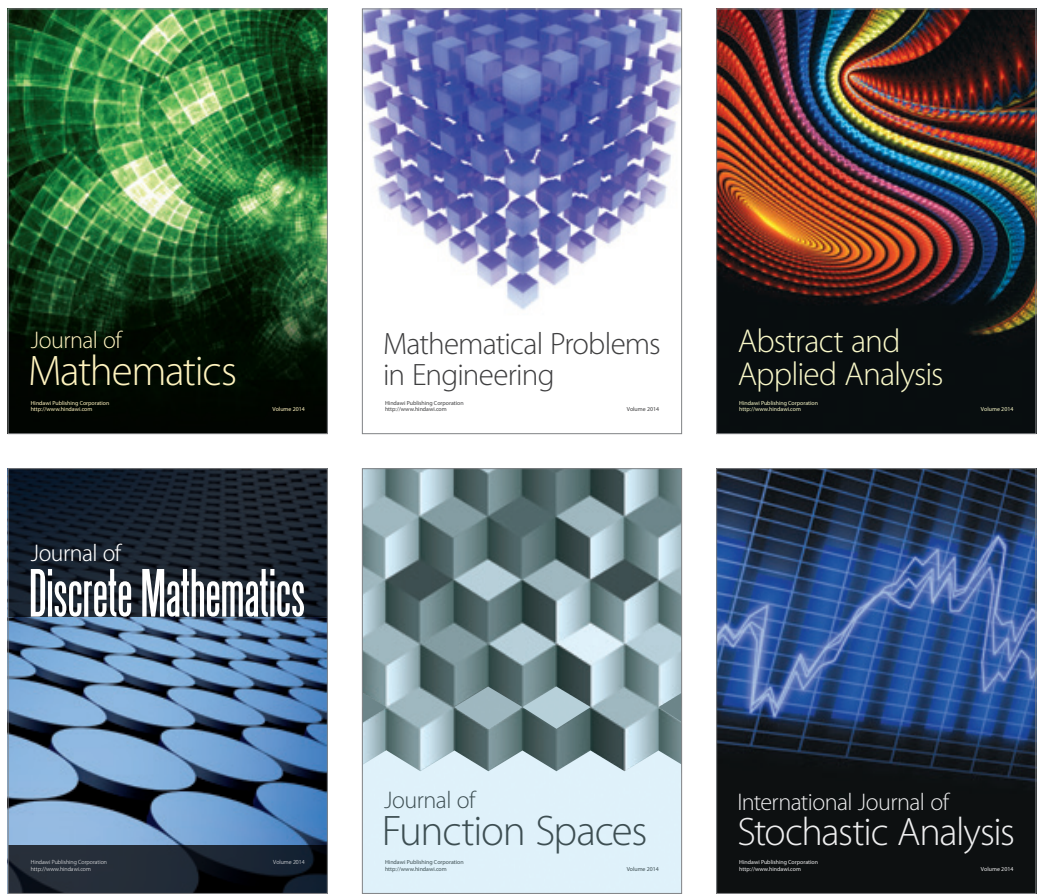

Journal of

Function Spaces

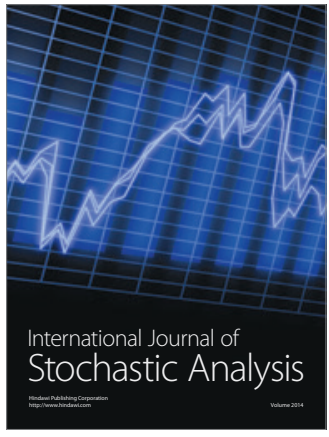

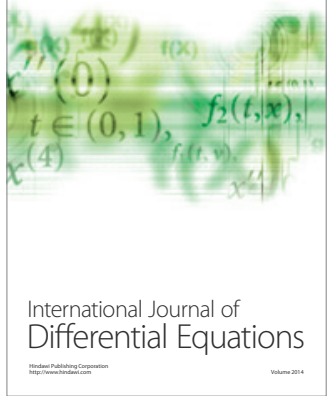
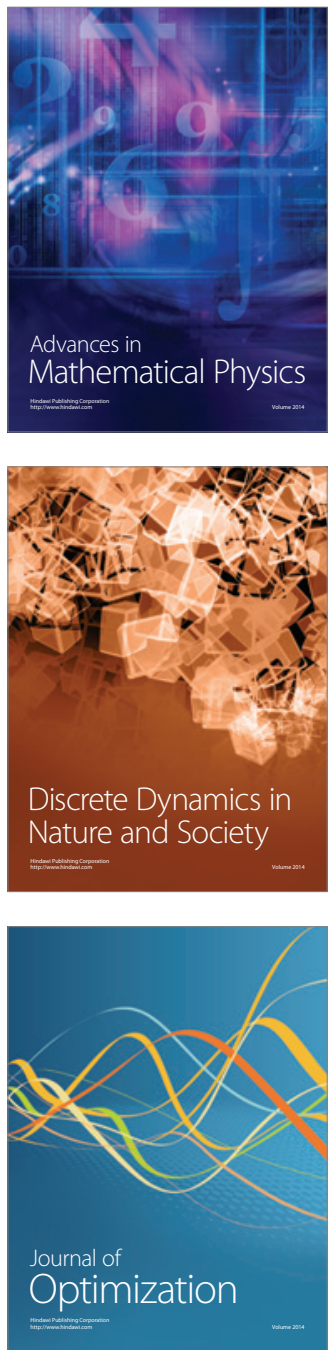